\title{
Estimation of Parameter for Different Magnetorheological Fluids Model for Varying Current
}

\author{
M. Khusyaie M. Razali, Asan G. A. Muthalif*, N. Hidayati Diana Nordin \\ Smart Structures, Systems and Control Research Laboratory (S $\left.{ }^{3} C R L\right)$, Department of Mechatronics \\ Engineering, Kulliyyah of Engineering, International Islamic University Malaysia (IIUM), Jalan Gombak, \\ 53100, Kuala Lumpur, Malaysia. \\ * Corresponding author. Email: asan@iium.edu.my \\ Manuscript submitted September16, 2017; accepted December 1, 2017. \\ doi: 10.17706/ijcee.2018.10.2.127-134
}

\begin{abstract}
Magnetorheological fluids (MRF) are considered as smart material since the behavior of the fluid is interrelated with external magnetic field. The rheological behavior of the fluids is changed whenever the strength of magnetic field changed. One of the applications of MRF is Magnetorheological damper (MRD), a damper that can be controlled by magnetic field. Analytical model of the damper is important in order to understand the performance of MRD. Different types of model that depend on defined parameters were introduced by previous researchers, and investigation on those parameters is crucial to acquire a model with least value of error. A technique called nonlinear least squares fitting method is utilized in order to obtain those parameters. This study investigated five different MRD models and presents the result for parameter estimation for each model as a function of input current.
\end{abstract}

Key words: Magnetorheological damper, magnetorheological fluids, MRF model, parameter estimation.

\section{Introduction}

Magnetorheological (MR) damper has been regarded as one of the most promising latest technologies. MR damper implemented the usage of special fluid called Magnetorheological Fluid (MRF) where the rheological properties of this fluid can be controlled by external magnetic field [1]. In normal condition where no magnetic field is present, the behavior of MRF is in free flowing fluid state. At this state, MRF exhibits Newtonian-like behavior. The behavior of MRF changes from free-flowing fluid to semi-solids with controllable yield strength whenever the external magnetic field is present [2]. When an external magnetic field is present, the MRF particle will form linear chains parallel to the field due to the dipole moment of particle aligned with the magnetic field. This will solidify the MRF and restrict the movement of the fluid [3].

Few types of model were developed to represent the behavior of MR damper. Some of the famous models are the Bingham model, Simple Bouc-Wen model, Modified Bouc-Wen model, Hyperbolic Tangent Function model and Nonlinear Biviscous model [1]. To design an optimal MR damper model so that the behavior of the model is most identical with the behavior of the MR damper, some reliable parameter values are needed [4]. Parameter estimation of an MR damper model with varying input current was done since the magnetic field is directly proportional to the current [5]. However, there is not much empirical study on the parameter estimation with varying input current for all the models mentioned above[6]-[8]. This research is focused on parameter estimation with varying input current for the five different models. 


\section{Magnetorheological (MR) Damper Model}

\subsection{Bingham Model}

Bingham model is proposed based on the rheological behavior of MR fluids [9]. The damping force of the Bingham model can be referred to (1).Frictional force, $f_{c}$ is related to the fluid yield stress that depends on the applied field, while offset force, $f_{o}$ is a stored energy or accumulator's effect produced from the excitation of the disturbance. $c_{o}$ is damping coefficient, $\dot{x}$ is the input velocity and $F$ is the output force measured from the MR damper [9]. Bingham model however, only considers the behavior of the MR fluid in the post-yield region but not in the pre-yield region where the fluids assumed to be rigid. Thus, Bingham model is not suitable to be used for dynamic application due to the lack in describing the fluid's properties at low shears rates and small deformations [10].

$$
F=c_{o} \dot{x}+f_{c} \operatorname{sgn}(\dot{x})+f_{o}
$$

\subsection{Simple Bouc-Wen Model}

Equation (2) is the damping force for the Simple Bouc-Wen model. $\alpha$ is a scaling value of MR damper related to material yield stress [11], $c_{o}$ and $k_{o}$ are damping coefficient and spring stiffness respectively, and $x_{o}$ is initial displacement of MR damper due to accumulator effect [1]. Evolutional variable $z$ is hysteretic deformation of the damper. $A, \beta, \gamma$ and $n$ are Bouc-Wen parameters to control the shape of the hysteresis loop. Additionally, those parameters are also used to control the smoothness of the transitions from pre-yield to post-yield region [7].The prediction of simple Bouc-Wen model is better compared to Bingham model since it better resembles the behavior of the damper. Still, this model does not totally represent the MR damper since it cannot describe the behavior of the nonlinear force-velocity response at yield region [12].

$$
\begin{gathered}
F=\alpha z+c_{o} \dot{x}+k_{o}\left(x-x_{o}\right) \\
\dot{z}=\gamma|\dot{x}| z\left(\left.z\right|^{n-1}\right)-\beta \dot{x}|z|^{n}+A \dot{x}
\end{gathered}
$$

\subsection{Modified Bouc-Wen Model}

Equation (4) is the damping force for the Modified Bouc-Wen model. $y$ is the internal displacement of damper [1]. $k_{1}$ represents as accumulator stiffness of the damper with $x_{0}$ as initial displacement due to the accumulator effect [12]. $c_{0}$ and $c_{1}$ are damping coefficients at large velocity and low velocity, respectively. This modified model improves the accuracy of estimation of MR damper behavior compared to the Simple Bouc-Wen Model [1]; however another problem arises due to difficulty in estimating the extended number of model coefficients. This problem is happened due to the assumption of linear relationship between model coefficient and the applied current [12]. Nonlinear relationship between applied current and unknown model coefficients should be implemented for better estimation of the model coefficients [13].

$$
\begin{gathered}
F=c_{1} y+k_{1}\left(x-x_{o}\right) \\
\dot{y}=\frac{1}{c_{o}+c_{1}}\left[\alpha z+c_{o} \dot{x}+k_{o}(x-y)\right] \\
\dot{z}=-\gamma|\dot{x}-\dot{y}||z|^{n-1} z-\beta(\dot{x}-\dot{y})|z| n+A(\dot{x}-\dot{y})
\end{gathered}
$$

\subsection{Hyperbolic Tangent Function Model}


Equation (7) is the damping force for the Hyperbolic Tangent Function model. $c_{0}$ and $k_{0}$ are damping and spring coefficients respectively. Effect of the accumulator of the damper is represented as offset force, $f_{0 .} \alpha$ is the scale factor of the hysteresis and $z$ is the hysteretic variable. $B$ and $\delta$ in hysteretic variable are damper parameters in controlling the shape of the hysteresis loop. $\beta$ is the scale factor in controlling the hysteretic slope and $\delta$ is the scale factor in controlling the width of the hysteresis loop[6]. A constant $\alpha$ is used to control the height of the hysteresis and offset force is used to shift the whole hysteresis loop. This model is better from the perspective of controller design, parameter identification and implementation [13].

$$
\begin{gathered}
F=c_{o} \dot{x}+k_{o} x+\alpha z+f_{o} \\
z=\tanh [\beta \dot{x}+\delta \operatorname{sign}(x)]
\end{gathered}
$$

\subsection{Nonlinear Biviscous Model}

This model assumes that MR fluids are behaved as plastic in both pre-yield and post-yield region. $c_{\text {pre }}$ and $c_{\text {post }}$ are the damping coefficient for pre-yield and post-yield respectively. $c_{\text {pre }}$ is always bigger than $c_{\text {post }}$ $\left(c_{\text {pre }}>c_{\text {post }}\right)$ which can be referred from Fig. $1 . F_{\mathrm{y}}$ is the yield force while $\dot{x}_{\mathrm{y}}$ is the yield velocity. The disadvantage of this model is its disability to perform well with varying input excitation current [1].

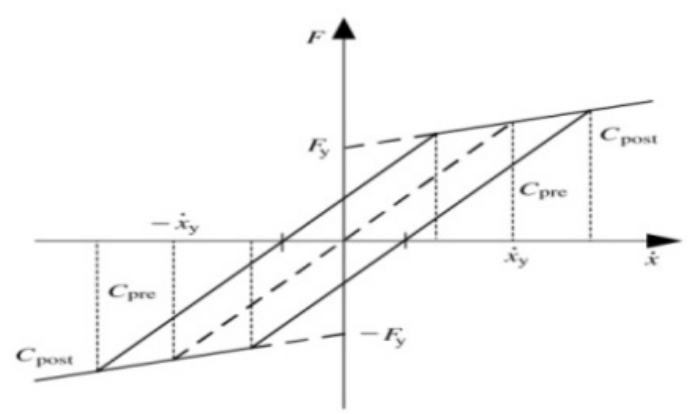

Fig. 1. Graph of MR damper behaviorfor the Nonlinear Biviscous model[1].

$$
\begin{aligned}
& F=\left\{\begin{array}{cc}
c_{\text {post }} \dot{x}+F_{y}, \quad & \dot{x} \geq \dot{x}_{y} \\
c_{\text {pre }} \dot{x}, & -\dot{x}_{y} \geq x \geq \dot{x}_{y} \\
c_{\text {post }} \dot{x}-F_{y}, \quad & \dot{x} \leq-\dot{x}_{y}
\end{array}\right. \\
& \dot{X}=\frac{F_{y}}{c_{\text {pre }}-c_{\text {post }}} c_{\text {pre }}
\end{aligned}
$$

\section{Parameter Estimation for Varying Current}

This section is to estimate the parameter of MR damper model by comparing the model with experimental result. LORD RD-8041-1 damper is tested by using Universal Testing Machine (UTM). The force applied on the damper is in downward motion and the experiment is repeated for a few times with different value of input current. The experimental setup with MR damper and force response from the test is shown in Fig. 2. Note that, three components are kept constant which are input piston velocity $(\dot{x}=500$ $\mathrm{mm} /$ minute), $n$ parameter $(n=2)$ and initial displacement of piston $\left(x_{o}=37 \mathrm{~mm}\right)$. Input current applied on the damper are from 0 to 1 Ampere. Nonlinear least square fitting method is used for parameter estimation using MATLAB[14]. The parameter is fitted with different input current, and errors from all models are compared to determine the most suitable model to represent the behavior of MR damper. 


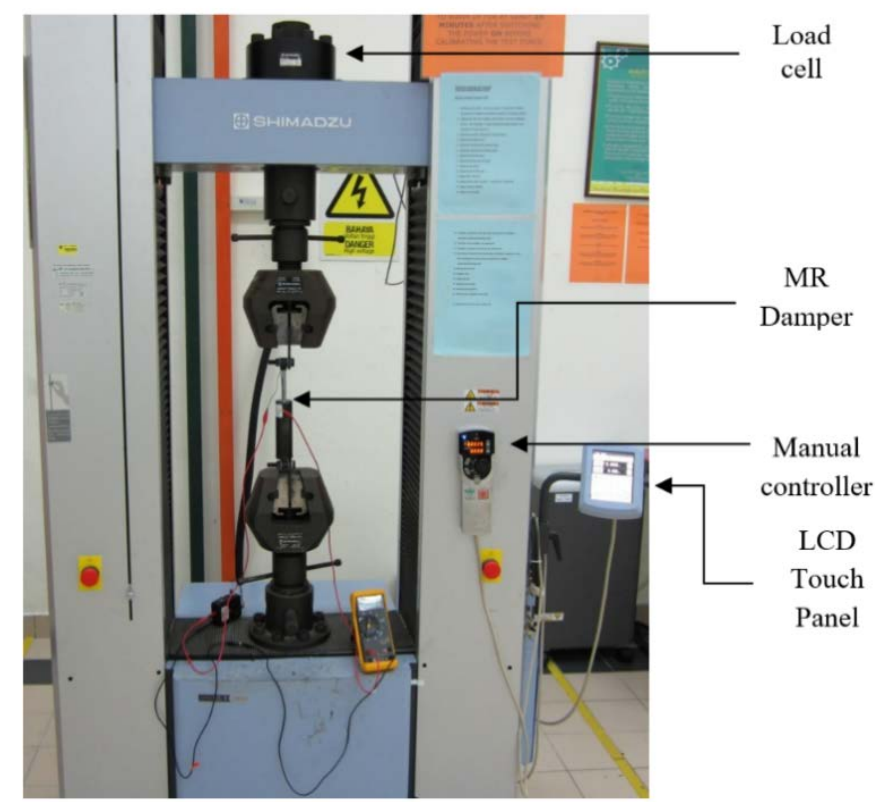

(a)

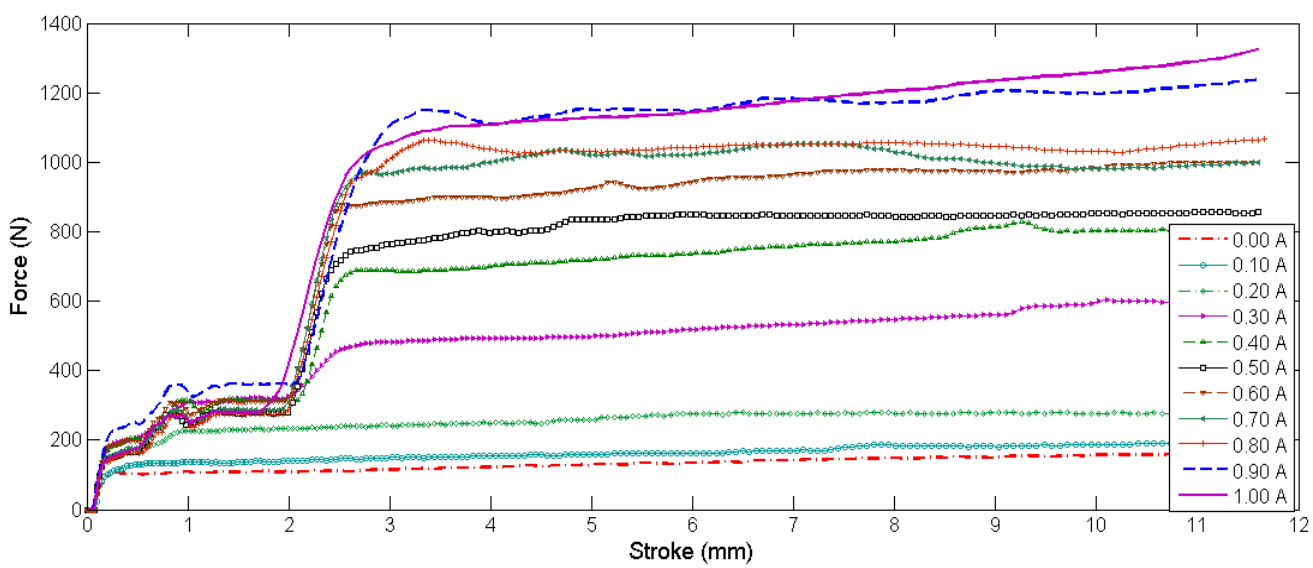

(b)

Fig. 2. (a) Experimental setup with MR damper, (b) Graph of damping forces from the experiment.

The parameter estimation equations for five models can be referred to Table 1 and the estimation errors are summarized in Table 2. The curve fitting graph between model parameter and input current can be referred to Fig. 3. The relationship between model parameter and input current is investigated in order to know the pattern between model parameter and varying input current. The result showed that the relationship between parameter value and input current mostly is nonlinear. This is due to the nonlinear behavior of MR damper itself. The parameter value also can be estimated directly from the estimated equations within 0 to $1 \mathrm{~A}$ of input current.

Table 1. Table of Parameter Estimation Equation

\begin{tabular}{|c|c|}
\hline Bingham model & Nonlinear Biviscous model \\
$c_{o}=147.5 i+13.52$ & $c_{2}=12.3$ \\
$f_{c}=-394.8 i+38.14$ & $c_{o}=-148.2 i^{3}+132.2 i^{2}+139.5 i+5.166$ \\
$f_{o}=-3.93 \times 10^{-08} i^{3}+4.43 \times 10^{-08} i^{2}-1.16 \times 10^{-08} i$ & $f_{c}=643.1 i^{3}-819.4 i^{2}-48.13 i+35.43$ \\
$+1.687 \times 10^{-10}$ & \\
\hline
\end{tabular}




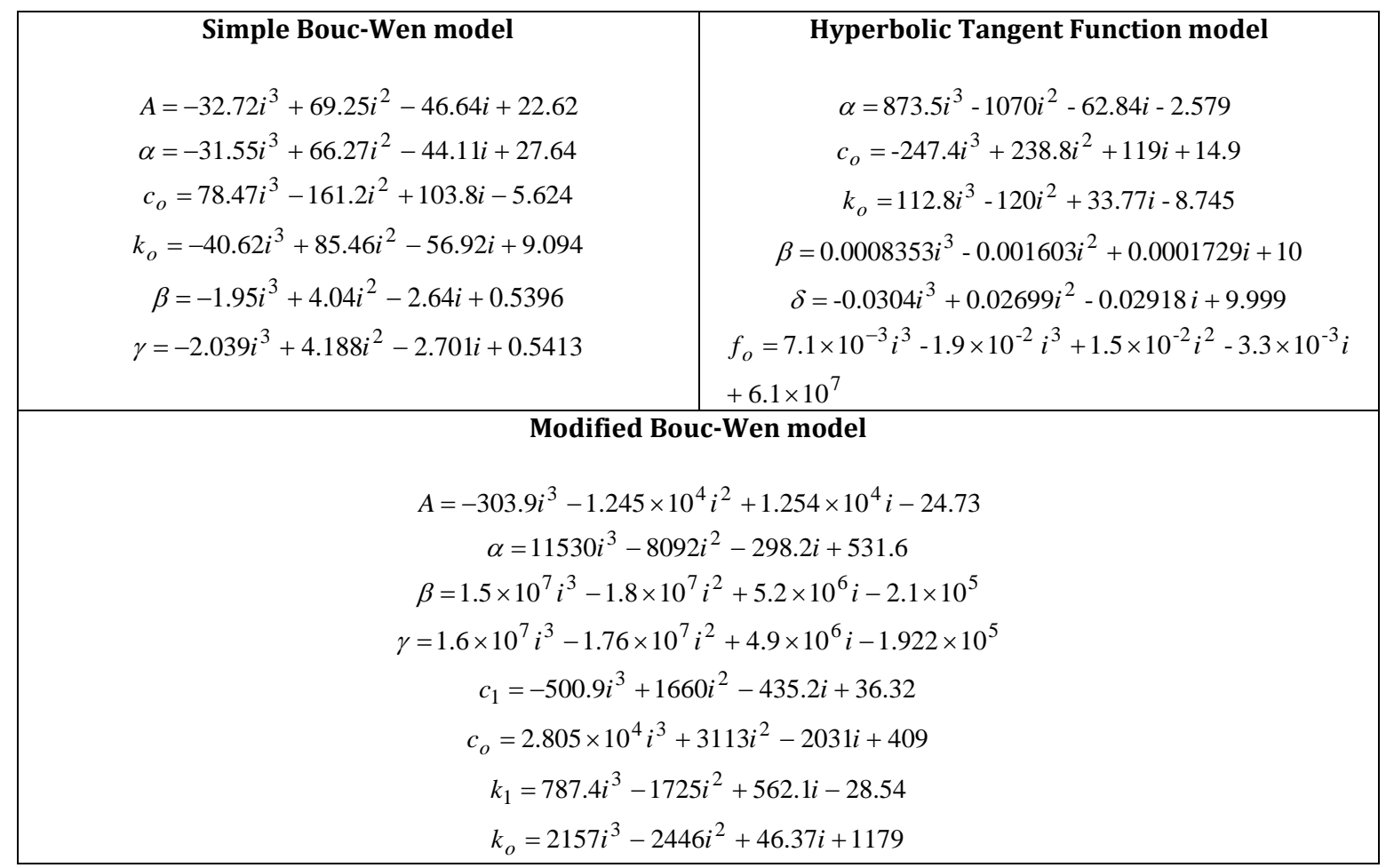

The precision of the curve fitting technique represents as R-squared. The determination ranges of R-squared are from zero to one. The regression line perfectly fits the data if and only if R-squared is one. The curve fitting result for all models are considered perfectly fit since the value of R-squared for each model is almost equal to one (Table 2).

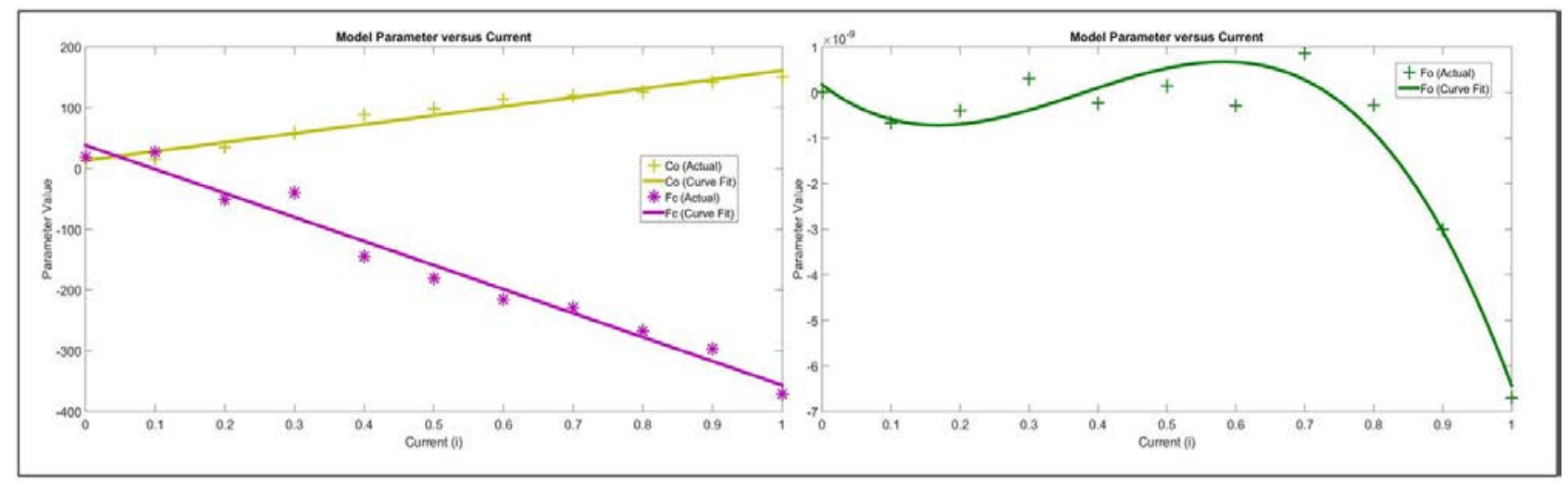

(a)

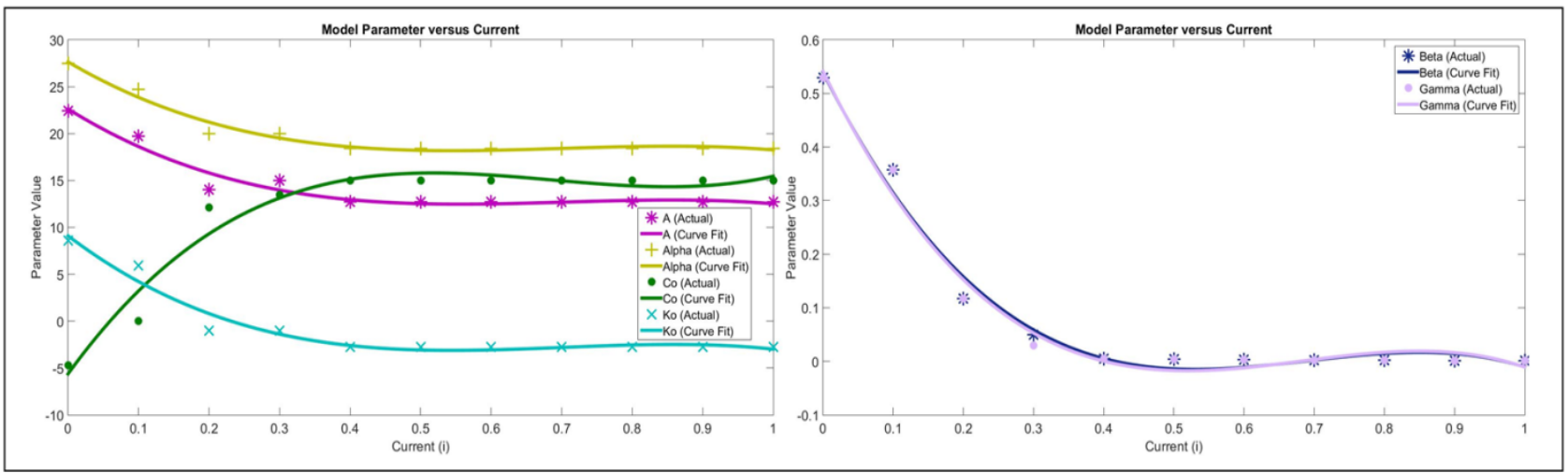

(b) 


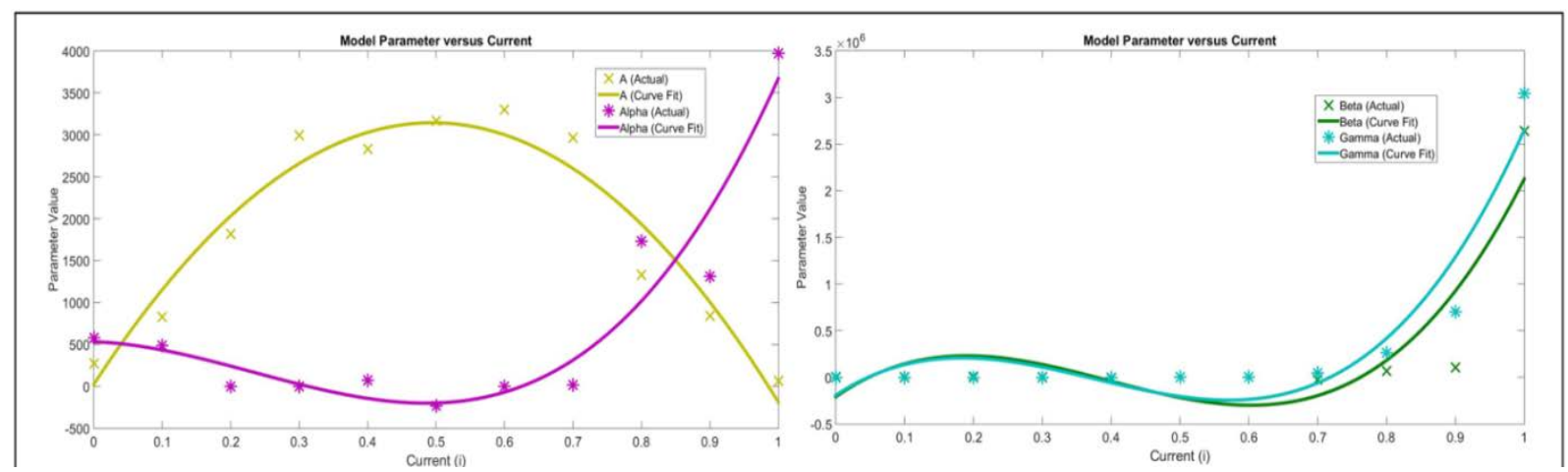

(c)

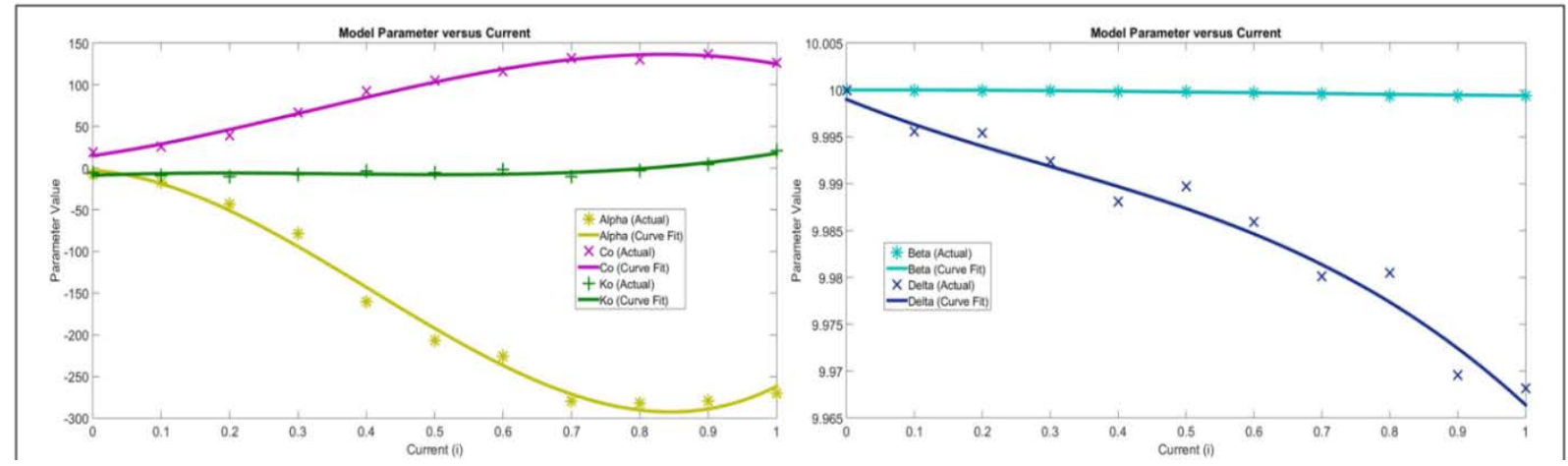

(d)

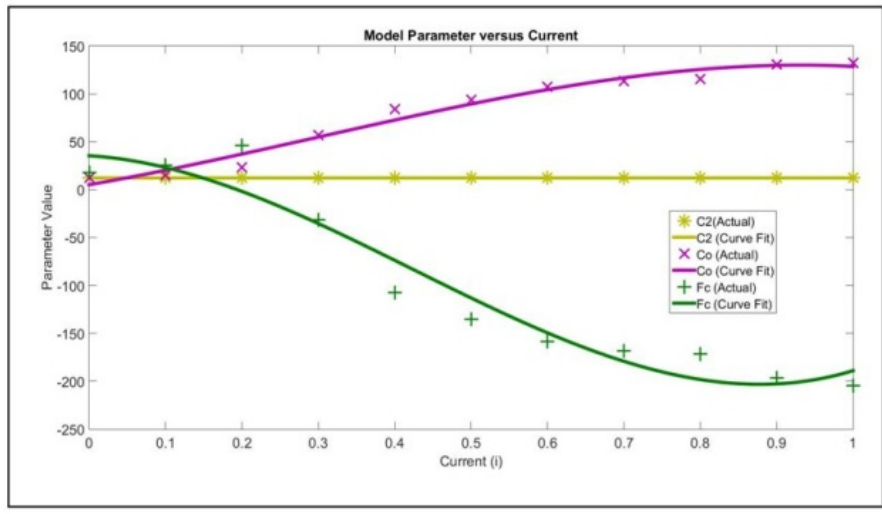

(e)

Fig. 3. Graph of curve fitting between model parameters and input current for (a) Bingham model; (b) Simple Bouc-Wen model; (c) Modified Bouc-Wen model; (d) Hyperbolic tangent function model; and (e) Nonlinear biviscous model.

Table 2. Table of R-Squared Value and Parameter Estimation Error

\begin{tabular}{|c|c|c|}
\hline MR Damper Model & R-Squared Value & Parameter Estimation Error (E) \\
\hline Bingham & 0.9595 & 3.8096 \\
\hline Simple Bouc Wen & 0.9622 & 1.9245 \\
\hline Modified Bouc-Wen & 0.8956 & 1.3770 \\
\hline Hyperbolic Tangent Function & 0.9327 & 3.3688 \\
\hline Nonlinear Biviscous & 0.9710 & 3.8052 \\
\hline
\end{tabular}

Selection of optimal model is important in order to represent the real behavior of MR damper in mathematical form which will be used in controller design. Better controller design due to better MR damper model selection will improve the performance of the MR damper. Selection of the model is done 
based on the parameter estimation error value, $E$. The lower the error value is, the closer the behavior of the model with the real one. Overall, the parameter estimation for all five MR damper models are satisfied since the parameter estimation error, $E$ is small where the maximum one is only 3.8096. From Table 2, Modified Bouc-Wen model is the most suitable model to represent the behavior of MR damper since the estimation error is the lowest.

\section{Conclusion}

Five parametric models of MR damper are discussed; Bingham model, Simple Bouc-Wen model, Modified Bouc-Wen model, Hyperbolic Tangent Function model and Nonlinear Biviscous model. Each model is analyzed by using nonlinear least square fitting method in order to obtain the parameter value. Each parameter is fitted with varying input current and the trend of the graph is observed to be nonlinear. The MR damper models are compared to each other in order to select the most optimal one. Based on the parameter estimation error value, Modified Bouc-Wen model is the optimum model since the error value is the lowest. This result can be used to design controller or simulation study.

\section{Acknowledgements}

This work is supported by Ministry of Higher Education Malaysia under the Exploratory Research Grant Scheme (ERGS13-020-0053).

\section{References}

[1] Kasemi, B., Muthalif, A. G. A., Rashid, M. M., \& Rahman, M. (2011, May). Optimizing dynamic range of magnetorheological fluid dampers : Modeling and simulation. Proceedings of International Conference on Mechatronics (pp. 17-19).

[2] Ferdaus, M. M. (2014). Temperature effect analysis on magneto-rheological damper's performance. Journal of Automation \& Control Engineering, 2(4), 392-396.

[3] Acocella, G., Anchini, R., Paciello, V., Pietrosanto, A., \& Sommella, P. (2010). A new approach to magnetorheological damping control. Proceedings of 2010 IEEE Int. Instrum. Meas. Technol. Conf. I2MTC 2010 (pp. 908-912).

[4] Truong, D., \& Ahn, K. (2012). MR fluid damper and its application to force sensorless damping control system. Smart Actuation Sens. Syst. - Recent Adv. Futur. Challenges, 383-424,

[5] Knoepfel, H. E., \& Mattis, D. C. (2001). Magnetic fields: A comprehensive theoretical treatise for practical use. American Journal of Physics, 69(4), 525.

[6] Kwok, N. M., Ha, Q. P., Nguyen, T. H., Li, J., \& Samali, B. (2006). A novel hysteretic model for magnetorheological fluid dampers and parameter identification using particle swarm optimization. Sensors Actuators A Phys., 132(2), 441-451.

[7] Braz-césar, M. T., \& Barros, R. C. (2013, June). Experimental and numerical analysis of mr dampers. Eccomasproceedings Org., 12-14.

[8] Yang, G. S., Carlson, J. D., \& Sain, K. (2002). Large-scale MR uid dampers: Modeling and dynamic performance considerations. Eng. Struct., 24(3), 309-323.

[9] Stanway, R., Sproston, J. L., \& Stevens, N. G. (1987, Dec.). Non-linear modelling of an electro-rheological vibration damper. J. Electrostat., 20(2), 167-184.

[10] Kamath, G. M., \& Wereley, N. M. (1999). A nonlinear viscoelastic - plastic model for electrorheological fluids. Smart Mater. Struct., 6(3), 351-359.

[11] Talatahari, S., Kaveh, A., \& Rahbari, N. M. (2012). Parameter identification of Bouc-Wen model for MR fluid dampers using adaptive charged system search optimization. Journal of Mechanical Science \& 
Technology, 26(8), 2523-2534.

[12] Spencer, B. F. J., Dyke, S. J., Sain, M. K., \& Carlson, J. D. (1997). Phenomenological model for magnetorheological dampers. J. Eng. Mech., 123(3), 230-238.

[13] Wang, D. H., \& Liao, W. H. (2011). Magnetorheological fluid dampers: A review of parametric modeling. Smart Mater. Struct., 20(2), 023001.

[14] Chen, K. F. (2010). Estimating parameters of a sine wave by separable nonlinear least squares fitting. IEEE Trans. Instrum. Meas., 59(12), 3214-3217.

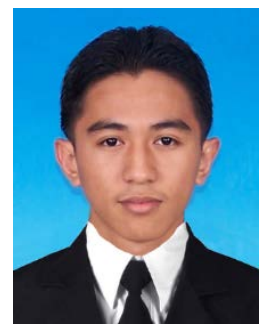

M. Khusyaie M. Razali received his bachelor degree in mechatronics engineering from International Islamic University Malaysia (IIUM), in 2015. He is currently working toward masters in mechatronics engineering in IIUM. His current research interest are on magnetorheological fluid damper and robust control.

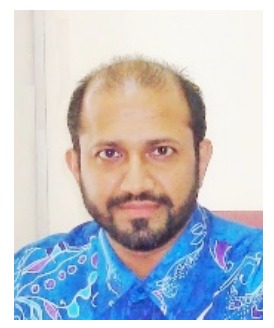

Asan G.A. Muthalif has obtained his bachelor degree and masters in mechatronics engineering from IIUM. He earned PhD from University of Cambridge, UK in 2008. Upon completing $\mathrm{PhD}$, he had continued post-doctoral research for a year in Cambridge University. His research interests are mechatronics, active \& semi-active vibration control, smart materials and structures, vibration based energy harvesting, mid-high frequency vibration control, statistical energy analysis (SEA), dynamics of built-up structures, and bio-mechatronics systems.

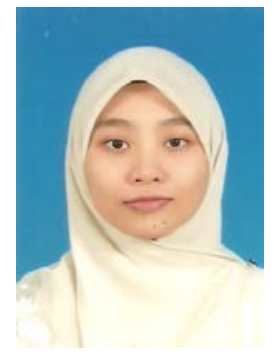

N.H. Diyana Nordin obtained her bachelor's and master's degrees in mechatronics engineering from IIUM. Her research interests include piezoelectric energy harvesting and magnetorheological fluid damper. She is currently pursuing her $\mathrm{PhD}$ in engineering at IIUM. 